\title{
[1] Purification and Biochemical Properties of Rac1, 2, 3 and the Splice Variant Rac1b
}

\author{
By Lars Christian Haeusler, lars Hemsath, Dennis Fiegen, \\ Lars Blumenstein, Ulrike Herbrand, Patricia Stege, \\ Radovan Dvorsky, and Mohammad Reza Ahmadian
}

\begin{abstract}
Rac proteins (Rac1, 1b, 2, 3) belong to the GTP-binding proteins (or GTPases) of the Ras superfamily and thus act as molecular switches cycling between an active GTP-bound and an inactive GDP-bound form through nucleotide exchange and hydrolysis. Like most other GTPases, these proteins adopt different conformations depending on the bound nucleotide, the main differences lying in the conformation of two short and flexible loop structures designated as the switch I and switch II region. The three distinct mammalian Rac isoforms, Rac1, 2 and 3, share a very high sequence identity (up to $90 \%$ ), with Rac1b being an alternative splice variant of Rac1 with a 19 amino acid insertion in vicinity to the switch II region. We have demonstrated that Rac1 and Rac3 are very closely related with respect to their biochemical properties, such as effector interaction, nucleotide binding, and hydrolysis. In contrast, Rac2 displays a slower nucleotide association and is more efficiently activated by the Rac-GEF Tiam1. Modeling and normal mode analysis corroborate the hypothesis that the altered molecular dynamics of Rac2, in particular at the switch I region, may be responsible for different biochemical properties. On the other hand, our structural and biochemical analysis of Rac1b has shown that, compared with Rac1, Rac1b has an accelerated GEF-independent GDP/GTP-exchange and an impaired GTP-hydrolysis, accounting for a self-activating GTPase. This chapter discusses the use of fluorescence spectroscopic methods, allowing real-time monitoring of the interaction of nucleotides, regulators, and effectors with the Rac proteins at submicromolar concentrations and quantification of the kinetic and equilibrium constants.
\end{abstract}

\section{Introduction}

The GTP-binding Rac-like proteins act as tightly regulated molecular switches that cycle between an inactive GDP-bound and an active GTP-bound state in response to a variety of extracellular stimuli. The interconversion between both states is controlled by two intrinsically 
slow biochemical reactions, namely the GDP/GTP exchange and the GTP-hydrolysis. Both reactions can be accelerated by several orders of magnitude by guanine nucleotide exchange factors (GEFs) and GTPase activating proteins (GAPs), respectively (Vetter and Wittinghofer, 2001). The crystal structures of several GTPases in either state revealed that the switching mechanism depends on the conformational change of two regions, termed switch I and switch II (Dvorsky and Ahmadian, 2004; Ihara et al., 1998). In the GTP-bound state, the switch regions provide a platform for the selective interaction with effector proteins (Bishop and Hall, 2000) and thereby initiate downstream signaling. The three distinct mammalian Rac isoforms (Rac1, 2 and 3) being encoded by different genes share between $89-93 \%$ amino acid sequence identity (Didsbury et al., 1989; Haataja et al., 1997; Polakis et al., 1989; Wherlock and Mellor, 2002). Rac1, the best-investigated isoform, regulates gene expression, cell cycle progression, and rearrangement of the actin cytoskeleton (Michiels and Collard, 1999). Rac2 is proposed to be responsible for the regulation of the oxidative burst in hematopoietic cells (Dinauer et al., 2003). Rac3 has been shown to be serum-inducible and hyperactive in breast cancer cells, where it controls proliferation by a PAK-dependent pathway (Haataja et al., 1997; Mira et al., 2000). Rac1 and Rac3 are ubiquitously expressed and therefore regulate a wide variety of cellular processes, whereas Rac2 is predominantly expressed in cells of the hematopoietic lineage. Rac1b was discovered in human tumors as an alternative splice variant of Rac1 containing a 19 amino acid insertion (between codons 75 and 76) next to the switch II region (Jordan et al., 1999; Schnelzer et al., 2000). It has been suggested that this insertion may create a novel effector-binding site in Rac1b and thus participates in signaling pathways related to the neoplastic growth of the intestinal mucosa (Jordan et al., 1999). Recently, it has been shown that Rac1b neither interacts with Rho-GDI, PAK1 nor induces lamellipodia formation but is able to promote growth transformation of NIH3T3 cells in a similar way as a constitutive active Rac1 mutant (Matos et al., 2003; Singh et al., 2004). In this chapter, we outline the methods that allowed us to quantitatively measure the physical interaction between the Rac proteins and nucleotides, GEFs, effectors and GAPs (Fiegen et al., 2004; Haeusler et al., 2003), providing a more detailed insight into the biochemical properties of the four mammalian Rac proteins (Table I).

Materials and Methods

\section{Buffers}

All buffers were filtered $(0.2 \mu \mathrm{m})$ and degassed. 
TABLE I

Biochemical Properties of Rac1, Rac1b, Rac2, and Rac3

\begin{tabular}{|c|c|c|c|c|}
\hline & Rac1 & Rac1b & Rac2 & Rac3 \\
\hline MantGDP-binding & $25 \times 10^{6}$ & $11 \times 10^{6}$ & $017 \times 10^{6}$ & $22 \times 10^{6}$ \\
\hline $\mathrm{k}_{\text {off }}\left(\mathrm{s}^{-1} ; \text { no Tiam } 1\right)^{a}$ & $0.7 \times 10^{-4}$ & $18 \times 10^{-4}$ & $1.2 \times 10^{-4}$ & $0.9 \times 10^{-4}$ \\
\hline $\mathrm{K}_{\text {off }}\left(\mathrm{s}^{-1} ; 5 \mu M \text { Tiam } 1\right)^{a}$ & $36 \times 10^{-4}$ & $19 \times 10^{-4}$ & $165 \times 10^{-4}$ & $32 \times 10^{-4}$ \\
\hline $\mathrm{K}_{\mathrm{d}}(\mathrm{p} M)$ & 28 & 1640 & 705 & 41 \\
\hline $\begin{array}{l}\text { PAK-GBD binding } \\
\mathrm{K}_{d}(\mu M ; 0-7.5 \mu M \text { GBD })^{b}\end{array}$ & 0.49 & $3.55^{d}$ & 0.13 & 0.61 \\
\hline $\begin{array}{l}\text { GTP-hydrolysis } \\
\text { rate }\left(\text { min }^{-1} ; \text { no GAP }\right)^{c} \\
\text { rate }\left(\min ^{-1} ; 8 \mu M \mathrm{GAP}\right)^{c}\end{array}$ & $\begin{array}{l}0.11 \\
2.36\end{array}$ & $\begin{array}{l}0.0035 \\
0.194\end{array}$ & $\begin{array}{l}0.16 \\
\text { n.d. }\end{array}$ & $\begin{array}{l}0.18 \\
\text { n.d. }\end{array}$ \\
\hline
\end{tabular}

The dissociation constant $\left(\mathrm{K}_{\mathrm{d}}\right)$ for the nucleotide binding values has been calculated from the association and the dissociation rate constants of the respective nucleotides $\left(\mathrm{K}_{\mathrm{d}}=\mathrm{k}_{\text {off }} /\right.$ $\left.\mathrm{k}_{\mathrm{on}}\right) .0 .1 \mu M^{a}, 0.2 \mu M^{b}$, and $80 \mu M^{c}$ Rac proteins were used for these measurements. ${ }^{d} \mathrm{Rac} 1 \mathrm{~b}$ measurements were carried out at $10^{\circ}$ due to the much faster nucleotide dissociation rate.

n.d.: not determined.

Buffer A: $30 \mathrm{~m} M$ Tris $/ \mathrm{HCl}, \mathrm{pH}$ 7.5, $1 \mathrm{~m} M \mathrm{MgCl}_{2}, 3 \mathrm{~m} M$ dithioerythritol (DTE), $50 \mathrm{mM} \mathrm{NaCl}$

Buffer B: $30 \mathrm{~m} M$ Tris/HCl, pH 7.5, $1 \mathrm{~m} M \mathrm{MgCl}_{2}, 10 \mathrm{~m} M \mathrm{Na}_{2} \mathrm{HPO}_{4} /$ $\mathrm{NaH}_{2} \mathrm{PO}_{4}, 3 \mathrm{~m} M$ DTE

Buffer C: $100 \mathrm{~m} M \mathrm{~K}_{2} \mathrm{HPO}_{4} / \mathrm{KH}_{2} \mathrm{PO}_{4}, \mathrm{pH} 6.5,10 \mathrm{~m} M$ tetrabutylammonium bromide, $7.5-25 \%$ acetonitrile.

\section{Proteins}

All proteins (i.e., Rac1, Rac1b, Rac2, Rac3), the catalytic domains of Tiam1 (DHPH; aa 1033-1404) and p50RhoGAP (GAP; aa 198-439), and the GTPase-binding domain (GBD) of $\alpha$ PAK (aa 57-141) were synthesized as glutathione-S-transferase (GST) fusion proteins in Escherichia coli. Bacterial lysates were applied to a glutathione-sepharose column (Amersham Pharmacia, Freiburg), and the GST-fusion proteins were eluted in buffer A containing $20 \mathrm{mM}$ glutathione. Cleavage of GST was performed in batch by incubation with 1-2 units of thrombin (Serva) per milligram proteins for $4-16 \mathrm{~h}$ at $4^{\circ}$. Subsequently, the cleaved proteins were subjected to two additional chromatographic steps, a gel filtration (Superdex 75, Pharmacia) and a second glutathione-sepharose affinity chromatography, to obtain proteins of interest at high purity and to remove residual GST. 


\section{Gpp $(\mathrm{NH}) \mathrm{p}$-Bound and mantGpp(NH)p-Bound Rac Proteins}

$\mathrm{Gpp}(\mathrm{NH}) \mathrm{p}$-bound and mantGpp(NH)p-bound Rac proteins were prepared using the enzymatic activity of alkaline phosphatase (Roche Diagnostics), thus degrading bound GDP. Herein, 0.1-1 of the enzyme were incubated overnight at $4^{\circ}$ with $1 \mathrm{mg}$ GDP-bound GTPase in buffer A containing a 1.2 molar excess of $\mathrm{Gpp}(\mathrm{NH}) \mathrm{p}$ or mantGpp(NH)p, $200 \mathrm{mM}$ ammonium sulfate, and $1 \mathrm{mM} \mathrm{ZnCl}$. The GDP degradation can be readily monitored by high-performance liquid chromatography (HPLC) analysis. After complete GDP degradation, the protein solution was applied to a prepacked NAP-5 gel filtration column (Pharmacia, Uppsala, Sweden) in buffer B. The concentration of the (mant-)Gpp(NH)p-bound GTPase was determined by HPLC as described later. The proteins were stored at $-80^{\circ}$.

\section{Preparation of the Nucleotide-Free GTPases}

Preparation of the nucleotide-free GTPases was carried out in two steps as originally described for H-Ras (John et al., 1990). In the first step, the bound GDP was degraded by alkaline phosphatase and replaced by $\mathrm{Gpp}(\mathrm{CH} 2) \mathrm{p}$ (another non-hydrolyzable GTP-analog, which is resistant to alkaline phosphatase) under the same conditions as described previously for the preparation of $\mathrm{Gpp}(\mathrm{NH}) \mathrm{p}$-bound GTPases. After GDP was completely degraded, 1-2 snake venom phosphodiesterase (SigmaAldrich) per milligram GTPase was added to the solution to cleave $\mathrm{Gpp}(\mathrm{CH} 2) \mathrm{p}$ between the $\alpha$ - and $\beta$-phosphate. The reaction was monitored by HPLC measurements, and after complete degradation of $\mathrm{Gpp}(\mathrm{CH} 2) \mathrm{p}$, both enzymes (alkaline phosphatase and phosphodiesterase) were inactivated by two cycles of snap freezing in liquid nitrogen and quick defrosting. In the presence of GMP or guanosine (the products of the enzymatic reactions), most GTPases are quite stable and can be stored at $-80^{\circ}$ for several months.

\section{MantGDP-Bound GTPases}

Fluorescent GDP-bound GTPases were prepared by loading nucleotide-free proteins with a 1.2-fold molar excess of mantGDP. Unbound nucleotides were separated from nucleotide-bound GTPases in buffer B on prepacked NAP-5 columns (Pharmacia). The concentrations of the respective nucleotide-bound GTPases were determined by HPLC as described later. The mantGDP-bound proteins were stored at $-80^{\circ}$. 


\section{Analysis of Free or Protein-Bound Fluorescent Nucleotides by Reversed-Phase HPLC}

Free- or protein-bound nucleotides (non-labeled or mant-labeled nucleotide) were analyzed by reversed-phase HPLC using buffer C, a C-18 column (ODS-Hypersil $5 \mu \mathrm{m}$, Bischoff, Leonberg, Germany), and a prefilter (Nucleosil $100 \mathrm{C} 18$, Bischoff), which separates protein-nucleotide complexes by adsorbing the denatured protein. A reasonable elution time ranging between 2 and 7 minutes on a C-18 column is achieved in buffer $\mathrm{C}$ containing $7.5 \%$ acetonitrile for non-labeled nucleotides and $25 \%$ acetonitrile for fluorescently labeled nucleotides. The HPLC system is calibrated with a solution having a clearly defined nucleotide concentration. The molar extinction coefficients at $252 \mathrm{~nm}$ of $13,700 \mathrm{M}^{-1} \mathrm{~cm}^{-1}$ for nonlabeled and $22,600 \mathrm{M}^{-1} \mathrm{~cm}^{-1}$ for mant-labeled guanine nucleotides were used. The linear relationship between peak area and nucleotide concentration allows calculating the concentration of nucleotide samples by integration of the respective peak areas.

\section{Fluorescence Spectroscopic Methods}

The stopped-flow instrument is routinely used for the analysis of rapid kinetics, such as guanine nucleotide association and GEF-catalyzed nucleotide dissociation (single turnover conditions) and the interaction between GTPases and their interacting partners (Ahmadian et al., 2002). The instrument (Applied Photophysics SX16MV) used had a fully automated operation modus and allowed collection of up to 1000 data points within a time window of $100 \mathrm{msec}$ up to $1000 \mathrm{sec}$. In the fully automated measuring step, equal volumes of two previously prepared solutions are rapidly injected into a mixing chamber where the fluorescence can be detected directly after the rapid mixing has occurred. The reaction time of the apparatus is approximately 2 msec. To obtain high accuracy, several identical measurements are recorded and averaged. For mant-nucleotides, the used excitation wavelength is $366 \mathrm{~nm}$, with the fluorescence being detected by a photomultiplier behind a cutoff-filter at wavelengths above $408 \mathrm{~nm}$. Reactions that are not completed within the 1000 -sec timeframe, such as nucleotide dissociation and indirect detection of GTPase-effector interactions using the guanine nucleotide dissociation inhibition (GDI) assay, can be monitored with a fluorescence spectrometer (Perkin-Elmer LS50B and SPEX Instruments FluoroMax II). With a temperature-controlled four-position turret, four experiments can be performed simultaneously, providing a better comparability of the single setups. The fluorescent component is provided in four 
quartz cuvettes (Hellma) that are thermally equilibrated until a constant fluorescence signal is reached. After addition of the reaction partner into the cuvette, a time-resolved fluorescence change can be monitored. For mantnucleotides, maximal fluorescence is obtained at excitation and emission wavelengths of 366 and $450 \mathrm{~nm}$, respectively.

Assays

\section{Nucleotide Binding}

The equilibrium dissociation constants $\left(\mathrm{K}_{\mathrm{d}}\right)$ of high-affinity interactions can be obtained by the kinetic approach from the ratio of the dissociation rate constant $\left(\mathrm{k}_{\mathrm{off}}\right)$ and the association rate constant $\left(\mathrm{k}_{\mathrm{on}}\right)$. Taking advantage of the large change in fluorescence intensity, the time course of the association of the nucleotide-free Rac proteins and the mant-labeled nucleotide can be monitored after rapidly mixing the two components in a stopped-flow instrument. The association of $0.1 \mu M$ mantGDP with increasing concentrations of nucleotide-free Rac $(0.5-10 \mu M)$ is measured in buffer B at $25^{\circ}$, which leads to an incremental increase in fluorescence intensity. The association kinetics exhibit a single exponential behavior under pseudo first-order conditions. The association rate constant $\left(\mathrm{k}_{\mathrm{on}}\right)$ is determined from the slope of the linear regression of the observed rate constants $\left(\mathrm{k}_{\mathrm{obs}}\right)$ plotted versus the concentration of nucleotide-free Rac. Because these experiments are performed under pseudo first-order conditions, the Rac proteins need to be in high molar excess. In principle, these experiments can be carried out for all other GTPases. However, the most important limiting factor is the accessibility and stability of the nucleotide free form of the respective GTPase. Measurements of the intrinsic nucleotide dissociation rate of the Rac proteins are carried out in a competitive displacement experiment by preincubating $0.1 \mu M$ mantGDP-bound Rac in buffer B at $25^{\circ}$ and a final cuvette volume of $600 \mu$ l. The reaction is initiated by adding a large molar excess of unlabeled GDP $(20 \mu M)$, leading to a very slow single exponential decrease in fluorescence. The presence of a large excess of unlabeled nucleotide ensures both a constant concentration for the exchange and a negligible signal of the reverse reaction, namely the reassociation of mantGDP. To make sure that the dissociation reaction has been completed, $20 \mathrm{~m} M$ EDTA was added to the sample, depleting Rac-bound magnesium ions and leading to a complete spontaneous nucleotide release. The exponential curve fitted to the obtained data yields the dissociation rate constant $\left(\mathrm{k}_{\text {off }}\right)$.

The dissociation constant $\left(\mathrm{K}_{\mathrm{d}}\right)$, calculated from the kinetic parameters of nucleotide dissociation and association reactions $\left(\mathrm{K}_{\mathrm{d}}=\mathrm{k}_{\mathrm{off}} / \mathrm{k}_{\mathrm{on}}\right)$ of 
the Rac proteins demonstrates the highest mantGDP affinity for Rac1 and the lowest for Rac1b with the following order Rac1 $>$ Rac3 $>$ Rac $2>\operatorname{Rac} 1 b$ (Table I). The overall affinity of Rac proteins for mantGDP is, particularly in the case of Rac1 and 3, in the same range as reported before for the members of other small GTPase families such as Ras (John et al., 1990), Ran (Klebe et al., 1995), and Rab (Simon et al., 1996). It is important to note that we obtained similar data for mantGTP binding to the nucleotidefree Rac proteins, suggesting that the presence of the $\gamma$-phosphate has no influence on nucleotide affinity.

\section{GEF-Catalyzed Nucleotide Dissociation (GEF) Assay}

A number of techniques are available for investigating the guanine nucleotide exchange of GTPases. A more detailed analysis of the interaction of the exchange factor with the GTPase-nucleotide complex is provided by fluorescence measurements in a stopped-flow instrument as described for Ran/RCC1 (Klebe et al., 1995), Ras/Cdc25 (Lenzen et al., 1998), Rap/C3G (van den Berghe et al., 1999), Rab/Vps9 (Esters et al., 2001), Rho/GDS (Hutchinson and Eccleston, 2000), and Rho/p190 (van Horck et al., 2001). The Tiam1-catalyzed mantGDP dissociation reaction of the Rac proteins is measured under the same conditions as described for the intrinsic mantGDP dissociation. These fast reactions are monitored in a stopped-flow instrument by rapid mixing of $0.1 \mu M$ Rac.mantGDP and $5 \mu M$ DHPH domain of Tiam1 with $20 \mu M$ GDP in buffer B at $25^{\circ}$. Using increasing GEF concentrations allows us to quantitatively determine the maximal GEF-catalyzed nucleotide dissociation rate $\left(\mathrm{k}_{\max }\right)$ and the apparent $K_{d}$ value for the interaction between GEF and nucleotide-bound Rac. A maximal Tiam1-activity of 6000-fold acceleration of intrinsic nucleotide dissociation was obtained for Rac2, which was 30-fold higher than that for Rac1 (Haeusler et al., 2003). We also have obtained an overall rate enhancement of 40,000-fold for the DHPH domain of the Rho-specific p190GEF (Haeusler et al., in preparation), suggesting that the $\mathrm{DH}$-containing proteins can be in principle very efficient GEFs.

\section{Effector-Induced Nucleotide Dissociation Inhibition (GDI) Assay}

GTPase-effector interactions can be quantitatively analyzed on the basis of the observation that effector GBDs bind close to the nucleotide binding region of the GTPase and, thereby, in contrast to the GEFs, inhibit the nucleotide dissociation (GDI effect). This method is used to determine the binding affinity of effector domains for their GTPases in an indirect manner, as described for Ras/Raf kinase (Herrmann et al., 1995, 1996), Ras/RalGDS (Linnemann, 2002; Rudolph et al., 1999), Ras/Byr2 (Scheffzek et al., 
2001), and Rho/Rhotekin, Rho/PKN, and Rho/Rho kinase interactions (Blumenstein and Ahmadian, 2004). Increasing concentrations of PAKGBD result in an incremental inhibition of the mantGppNHp dissociation from Rac, using $0.2 \mu M$ Rac.mantGppNHp and $40 \mu M$ GppNHp in buffer B at $25^{\circ}$. Individual observed rate constants $\left(\mathrm{k}_{\mathrm{obs}}\right)$ are calculated from the respective single exponential decay and plotted against the PAK concentrations to obtain the $\mathrm{K}_{\mathrm{d}}$ values according to an equation described by Hermann et al. $(1995,1996)$.

\section{Intrinsic and GAP-Stimulated GTP-Hydrolysis Reaction}

The intrinsic and GAP-stimulated GTP-hydrolysis reaction of the Rac proteins can be measured using various methods as previously described (Ahmadian et al., 2002). A generally useful and accurate method is HPLC. Hereby, $80 \mu M$ nucleotide-free GTPase and $70 \mu M$ GTP are incubated at $25^{\circ}$ in a final volume $200 \mu \mathrm{l}$ buffer B containing $10 \mathrm{mM} \mathrm{MgCl} 2$ in the absence and presence of $8 \mu M$ GAP. Samples of $25 \mu \mathrm{l}$ are taken at different time intervals and snap frozen in liquid nitrogen to halt the GTP-hydrolysis reaction. The samples $(25 \mu \mathrm{l})$ are then applied to a RP-18 HPLC column equilibrated with buffer $\mathrm{C}$ containing $7.5 \%$ acetonitrile and isocratically eluted at a flow rate of $1.8 \mathrm{ml} / \mathrm{min}$. Concentrations of GDP and GTP are determined from the area of the elution peaks of GTP and GDP to calculate the relative GTP content, represented by the ratio [GTP]/ $([\mathrm{GTP}]+[\mathrm{GDP}])$, to describe the reaction progress. By plotting the relative GTP content against the time, the hydrolysis rate $\mathrm{k}_{\text {cat }}$ can be determined with a single exponential fit of the data points.

Most recently, we synthesized a new fluorescent GTP, which is sensitive toward the GTP hydrolysis-induced conformational changes of the Rho GTPases and thus allows direct monitoring of the kinetics of the intrinsic and GAP-catalyzed GTP-hydrolysis reactions (Eberth et al., submitted).

\section{Biochemical Evaluation of the Rac Proteins}

The biochemical assays described previously together with structural studies have shown that the Rac isoforms exhibit different properties concerning ligand- and protein-protein interactions (Table I; Fiegen et al., 2004; Haeusler et al., 2003). Whereas Rac1 and Rac3 behave almost identically, Rac2 revealed (1) a 25 -fold lower nucleotide affinity because of a decreased nucleotide association rate, (2) a slightly higher PAK binding affinity, and (3) a significant increase in Tiam1-catalyzed nucleotide dissociation. These aberrant properties are the consequence of different conformational flexibilities in the switch I region (Haeusler et al., 2003). In 
contrast, Rac1b showed (1) a dramatic increase in its intrinsic nucleotide dissociation, which cannot be further enhanced by Tiam1; (2) a drastic decrease in intrinsic GTP-hydrolysis rate, which can be restored by GAP; and (3) reduced affinity for PAK-GBD. The altered characteristics of Rac1b are based on an open switch I conformation and a highly mobile switch II, which are induced by the 19 amino acid insertion (Fiegen et al., 2004).

\section{Acknowledgments}

Work in the authors' laboratory is supported, in part, by a European Community Marie Curie Fellowship, the Volkswagen-Stiftung, the Deutsche Forschungsgemeinschaft, the Max Planck Society, the Verband der Chemischen Industrie and the Bundesministerium für Bildung und Forschung.

\section{References}

Ahmadian, M. R., Wittinghofer, A., and Herrmann, C. (2002). Fluorescence methods in the study of small GTP-binding proteins. Methods Mol. Biol. 189, 45-63.

Bishop, A. L., and Hall, A. (2000). Rho GTPases and their effector proteins. Biochem. J. 348, 241-255.

Blumenstein, L., and Ahmadian, M. R. (2004). Models of the cooperative mechanism for Rhoeffector recognition: Implications for RhoA-mediated effector activation. J. Biol. Chem. 279, 53419-53426.

Didsbury, J., Weber, R. F., Bokoch, G. M., Evans, T., and Snyderman, R. (1989). Rac, a novel Ras-related family of proteins that are botulinum toxin substrates. J. Biol. Chem. 264, 16378-16382.

Dinauer, M. C. (2003). Regulation of neutrophil function by Rac GTPases. Curr. Opin. Hematol. 10, 8-15.

Dvorsky, R., and Ahmadian, M. R. (2004). Always look on the bright site of Rho-structural implications for a conserved intermolecular interface. EMBO Rep. 5, 1130-1136.

Esters, H., Alexandrov, K., Iakovenko, A., Ivanova, T., Thoma, N., Rybin, V., Zerial, M., Scheidig, A. J., and Goody, R. S. (2001). Vps9, Rabex-5 and DSS4: Proteins with weak but distinct nucleotide-exchange activities for Rab proteins. J. Mol. Biol. 310, 141-156.

Fiegen, D., Haeusler, L. C., Blumenstein, L., Herbrand, U., Dvorsky, R., Vetter, I. R., and Ahmadian, M. R. (2004). Alternative splicing of Rac1 creates a self-activating GTPase. J. Biol. Chem. 279, 4743-4749.

Haataja, L., Groffen, J., and Heisterkamp, N. (1997). Characterization of RAC3, a novel member of the Rho family. J. Biol. Chem. 272, 20384-20388.

Haeusler, L. C., Blumenstein, L., Stege, P., Dvorsky, R., and Ahmadian, M. R. (2003). Comparative functional analysis of the Rac GTPases. FEBS Lett. 555, 556-560.

Herrmann, C., Martin, G. A., and Wittinghofer, A. (1995). Quantitative analysis of the complex between p21ras and the Ras-binding domain of the human Raf-1 protein kinase. J. Biol. Chem. 270, 2901-2905. 
Herrmann, C., Horn, G., Spaargaren, M., and Wittinghofer, A. (1996). Differential interaction of the ras family GTP-binding proteins H-Ras, Rap1A, and R-Ras with the putative effector molecules Raf kinase and Ral-guanine nucleotide exchange factor. J. Biol. Chem. 271, 6794-6800.

Hutchinson, J. P., and Eccleston, J. F. (2000). Mechanism of nucleotide release from Rho by the GDP dissociation stimulator protein. Biochemistry 39, 11348-11359.

Ihara, K., Muraguchi, S., Kato, M., Shimizu, T., Shirakawa, M., Kuroda, S., Kaibuchi, K., and Hakoshima, T. (1998). Crystal structure of human RhoA in a dominantly active form complexed with a GTP analogue. J. Biol. Chem. 273, 9656-9666.

John, J., Sohmen, R., Feuerstein, J., Linke, R., Wittinghofer, A., and Goody, R. S. (1990). Kinetics of interaction of nucleotides with nucleotide-free H-ras p21. Biochemistry 29, 6058-6065.

Jordan, P., Brazao, R., Boavida, M. G., Gespach, C., and Chastre, E. (1999). Cloning of a novel human Rac1b splice variant with increased expression in colorectal tumors. Oncogene 18, 6835-6839.

Klebe, C., Prinz, H., Wittinghofer, A., and Goody, R. S. (1995). The kinetic mechanism of Ran-nucleotide exchange catalyzed by RCC1. Biochemistry 34, 12543-12552.

Lenzen, C., Cool, R. H., Prinz, H., Kuhlmann, J., and Wittinghofer, A. (1998). Kinetic analysis by fluorescence of the interaction between Ras and the catalytic domain of the guanine nucleotide exchange factor Cdc25Mm. Biochemistry 37, 7420-7430.

Linnemann, T., Kiel, C., Herter, P., and Herrmann, C. (2002). The activation of RalGDS can be achieved independently of its Ras binding domain. Implications of an activation mechanism in Ras effector specificity and signal distribution. J. Biol. Chem. 277, 7831-7837.

Matos, P., Collard, J. G., and Jordan, P. (2003). Tumor-related alternatively spliced Rac1b is not regulated by Rho-GDP dissociation inhibitors and exhibits selective downstream signaling. J. Biol. Chem. 278, 50442-50448.

Michiels, F., and Collard, J. G. (1999). Rho-like GTPases: Their role in cell adhesion and invasion. Biochem. Soc. Symp. 65, 125-146.

Mira, J. P., Benard, V., Groffen, J., Sanders, L. C., and Knaus, U. G. (2000). Endogenous, hyperactive Rac3 controls proliferation of breast cancer cells by a p21-activated kinasedependent pathway. Proc. Natl. Acad. Sci. USA 97, 185-189.

Polakis, P. G., Weber, R. F., Nevins, B., Didsbury, J. R., Evans, T., and Snyderman, R. (1989). Identification of the Ral and Rac1 gene products, low molecular mass GTP-binding proteins from human platelets. J. Biol. Chem. 264, 16383-16389.

Rudolph, M. G., Wittinghofer, A., and Vetter, I. R. (1999). Nucleotide binding to the G12Vmutant of Cdc42 investigated by X-ray diffraction and fluorescence spectroscopy: Two different nucleotide states in one crystal. Protein Sci. 8, 778-787.

Scheffzek, K., Grunewald, P., Wohlgemuth, S., Kabsch, W., Tu, H., Wigler, M., Wittinghofer, A., and Herrmann, C. (2001). The Ras-Byr2RBD complex: Structural basis for Ras effector recognition in yeast. Structure 9, 1043-1050.

Schnelzer, A., Prechtel, D., Knaus, U., Dehne, K., Gerhard, M., Graeff, H., Harbeck, N., Schmitt, M., and Lengyel, E. (2000). Rac1 in human breast cancer: Overexpression, mutation analysis, and characterization of a new isoform, Rac1b. Oncogene 19, 3013-3020.

Simon, I., Zerial, M., and Goody, R. S. (1996). Kinetics of interaction of Rab5 and Rab7 with nucleotides and magnesium ions. J. Biol. Chem. 271, 20470-20478.

Singh, A., Karnoub, A. E., Palmby, T. R., Lengyel, E., Sondek, J., and Der, C. J. (2004). Rac1b, a tumor associated, constitutively active Rac1 splice variant, promotes cellular transformation. Oncogene 23, 9369-9380. 
van den Berghe, N., Cool, R. H., and Wittinghofer, A. (1999). Discriminatory residues in Ras and Rap for guanine nucleotide exchange factor recognition. J. Biol. Chem. 274, 11078-11085.

van Horck, F. P., Ahmadian, M.R, Haeusler, L. C., Moolenaar, W. H., and Kranenburg, O. (2001). Characterisation of p190RhoGEF: A RhoA-specific guanine nucleotide exchange factor that interacts with microtubules. J. Biol. Chem. 276, 4948-4956.

Vetter, I. R., and Wittinghofer, A. (2001). The guanine nucleotide-binding switch in three dimensions. Science 294, 1299-1304.

Wherlock, M., and Mellor, H. (2002). The Rho GTPase family: A Racs to Wrchs story. J. Cell. Sci. 115, 239-240. 